\title{
An estimation for a family of oscillatory integrals
}

\author{
by \\ Magali Folch-Gabayet (México) and James Wright (Edinburgh)
}

\begin{abstract}
Let $K$ be a Calderón-Zygmund kernel and $P$ a real polynomial defined on $\mathbb{R}^{n}$ with $P(0)=0$. We prove that convolution with $K \exp (i / P)$ is continuous on $L^{2}\left(\mathbb{R}^{n}\right)$ with bounds depending only on $K, n$ and the degree of $P$, but not on the coefficients of $P$.
\end{abstract}

1. Introduction. In [5] Stein showed that if $P$ is a polynomial on $\mathbb{R}^{n}$ of degree $d$ with real coefficients and $K$ is a Calderón-Zygmund kernel, then

$$
\left|\int_{\mathbb{R}^{n}} e^{i P(x)} K(x) d x\right| \leq C_{d, n, K}
$$

where $C_{d, n, K}$ is independent of the coefficients of $P$ (the integral in (1) is taken as a principal value integral). This extends the corresponding onedimensional estimate due to Stein and Wainger [6].

As a consequence, if one defines the principal value tempered distribution $L$ by

$$
L(\phi)=\lim _{\varepsilon \rightarrow 0} \int_{\varepsilon \leq|x|} \phi(x) e^{i P(x)} K(x) d x, \quad \phi \in \mathcal{S},
$$

then the translation-invariant operator $f \mapsto f * L$ is bounded on $L^{2}\left(\mathbb{R}^{n}\right)$ uniformly over all polynomials of degree at most $d$. More generally if $Q$ : $\mathbb{R}^{n} \rightarrow \mathbb{R}^{m}$ is another polynomial, the same $L^{2}$ boundedness follows for convolution with the distribution

$$
\Lambda(\phi)=\text { p.v. } \int_{\mathbb{R}^{n}} \phi(Q(x)) e^{i P(x)} K(x) d x, \quad \phi \in \mathcal{S}\left(\mathbb{R}^{m}\right) .
$$

These $L^{2}$ bounds can be extended to $L^{p}$ bounds, $1<p<\infty$ (see [3] and [4]).

One cannot extend the estimate in (1) to phases which are general rational functions. For example, suppose $n=2$ and $K=\Omega(\theta) / r^{2}$ in polar coordinates. Then the oscillatory integral

$$
\int_{\mathbb{R}^{2}} e^{i y / x} K(x, y) d x d y=\int_{0}^{2 \pi} e^{i \tan \theta} \Omega(\theta) \int_{0}^{\infty} \frac{1}{r} d r d \theta
$$

2000 Mathematics Subject Classification: Primary 42B15. 
does not converge even as a principal value integral if $\int_{0}^{2 \pi} e^{i \tan \theta} \Omega(\theta) d \theta \neq 0$. For a counterexample where the principal value integral exists, one simply modifies the phase function to be $y /(1+x)$. A simple computation shows that

$$
\int_{x^{2}+y^{2} \leq N^{2}} e^{i y /(1+x)} K(x, y) d x d y=\log N \int_{0}^{2 \pi} e^{i \tan \theta} \Omega(\theta) d \theta+O(1) .
$$

When $n \geq 2$, similar examples to the ones considered above show that oscillatory integrals of the form $\int \exp (i P(x) / Q(x)) K(x) d x$ can never be bounded for general $K$ uniformly in the coefficients of $P$ and $Q$ if $P$ is allowed to vary over all polynomials of degree at most $d$ and $Q$ varies over polynomials of degree $d^{\prime}$ and both $d, d^{\prime} \geq 1$. This seems to be a higher dimensional phenomenon. We suspect the corresponding one-dimensional statement is true.

In this short note, we shall extend (1) to rational phases of the form $P(x)+1 / Q(x)$ where $P$ and $Q$ are real polynomials with $Q(0)=0$. We consider Calderón-Zygmund kernels $K(x)=\Omega(x) /|x|^{n}$ where $\Omega \in L \log L\left(\mathbf{S}^{n-1}\right)$ is homogeneous of degree zero with mean value zero, i.e., $\int_{\mathbf{S}^{n-1}} \Omega d \sigma=0$.

Theorem 1.1. With $K, P$ and $Q$ as above,

$$
\mid \text { p.v. } \int_{\mathbb{R}^{n}} e^{i(P(x)+1 / Q(x))} K(x) d x \mid \leq A
$$

where $A$ depends on $\|\Omega\|_{L \log L\left(\mathbf{S}^{n-1}\right)}$, n and the degrees of $P$ and $Q$ but not otherwise on the coefficients of $P$ and $Q$.

REMARKs 1.2. (i) Nagel and Wainger observed (see [5]) that for odd $K$, one could prove (3) from the corresponding one-dimensional version by the method of rotations.

(ii) As an immediate consequence of Theorem 1.1 we have

Corollary 1.3. With $K$ and $Q$ as in Theorem 1.1 but now $P$ : $\mathbb{R}^{n} \rightarrow \mathbb{R}^{m}$ being a polynomial mapping, convolution with the distribution

$$
L(\phi)=\text { p.v. } \int_{\mathbb{R}^{n}} \phi(P(x)) e^{i / Q(x)} K(x) d x, \quad \phi \in \mathcal{S}\left(\mathbb{R}^{m}\right),
$$

is bounded on $L^{2}\left(\mathbb{R}^{m}\right)$.

(iii) The requirement $Q(0)=0$ is most likely not necessary. For instance when the degree of $Q$ is 1 , the condition $Q(0)=0$ is easily removed. In fact:

Proposition 1.4. With $K$ and $P$ as in Theorem 1.1 but now $Q(x)=$ $a+v \cdot x$ where $a \in \mathbb{R}$ and $v \in \mathbb{R}^{n}$,

$$
\mid \text { p.v. } \int_{\mathbb{R}^{n}} e^{i(P(x)+1 / Q(x))} K(x) d x \mid \leq A
$$


where $A$ depends on $\|\Omega\|_{L \log L\left(\mathbf{S}^{n-1}\right)}, n$ and the degree of $P$ but not otherwise on $a, v$ and the coefficients of $P$.

When $n=1$, Proposition 1.4 gives uniform estimates for oscillatory integrals $\int \exp (i R(t)) d t / t$ where $R$ is a real rational function whose denominator has degree at most 1.

Notation. Let $A, B$ be complex-valued quantities. We use $A \lesssim B$ or $A=O(B)$ to denote the estimate $|A| \leq C_{\left(\|\Omega\|_{L \log L}, n, d, d^{\prime}\right)}|B|$ where $d$ and $d^{\prime}$ denote the degrees of $P$ and $Q$. We use $A \sim B$ to denote the estimate $A \lesssim B \lesssim A$.

In the following section we prove some preliminary lemmas needed for the proof of Theorem 1.1; the proof of Theorem 1.1 is then given in Section 3. Section 4 contains the proof of Proposition 1.4.

Acknowledgements. We would like to thank Tony Carbery for several clarifying discussions. The first author acknowledges financial support from CONACyT (32408-E) and DGAPA-UNAM (PAPIIT IN102799).

2. Preliminaries. The proof of Theorem 1.1 is similar to the proof of (1) given in [5] where one uses polar coordinates to reduce the integral to an integration over the unit sphere of an oscillatory integral in the radial variable. Stationary phase methods are used to estimate the radial integral reducing matters to sublevel estimates for homogeneous polynomials over the sphere $\mathbf{S}^{n-1}$.

More precisely, the estimate relies mainly on two lemmas. The first lemma gives an elementary decomposition of polynomials of one variable which is in the spirit of a decomposition in [2]. The second lemma is an integral estimate for homogeneous polymomials over the unit sphere due to Stein [5] which can be deduced from uniform sublevel set estimates for polynomials. To estimate one-dimensional oscillatory integrals, we use the well-known "van der Corput" lemma, which we state as the final lemma in this section.

LEMma 2.1. For any polynomial $Q(r)=\sum_{k=1}^{d} b_{k} r^{k}$ on $\mathbb{R}^{+}$, there is a finite collection $\left\{G_{j}\right\}_{j=1}^{M}$ of disjoint intervals, called "gaps", of $\mathbb{R}^{+}$with $M=O(1)$ such that

(1) The complement $\mathbb{R}^{+} \backslash \bigcup_{j=1}^{M} G_{j}$ is the union of $M-1$ disjoint "dyadic" intervals; that is, the ratio of the endpoints of such intervals is $\sim 1$.

(2) For each $G_{j}$, there is a $k=k_{j}, 1 \leq k_{j} \leq d$, such that for $r \in G_{j}$,

$$
|Q(r)| \sim\left|b_{k_{j}}\right| r^{k_{j}} \quad \text { and }\left|Q^{\prime}(r)\right| \sim\left|b_{k_{j}}\right| r^{k_{j}-1} \text {. }
$$
then

Also if $L_{j}$ and $R_{j}$ denote the left and right endpoints of $G_{j}$ respectively, 

and

(i) if $R_{j}<\infty$, then $R_{j}=C_{d}\left[\left|b_{l}\right| /\left|b_{m}\right|\right]^{1 / m-l}$ for some $1 \leq l<m \leq d$

(ii) if $L_{j}>0$, then $L_{j}=C_{d}\left[\left|b_{r}\right| /\left|b_{s}\right|\right]^{1 / s-r}$ for some $1 \leq r<s \leq d$.

Proof. We induct on the degree $d$. The case $d=1$ is trivial since then we have just one gap $G=(0, \infty)$ and no dyadic intervals.

Now suppose the lemma holds for all polynomials of degree $\leq d-1$ and write

$$
Q(r)=\sum_{i=1}^{d} b_{i} r^{i}=P(r)+b_{d} r^{d}, \quad b_{d} \neq 0 .
$$

We will construct the dyadic intervals for $Q$, the complementary intervals will be the gaps. Knowing the lemma holds for $P$ we can list its gaps: $G_{1}=\left[0, R_{1}\right], G_{2}=\left[L_{2}, R_{2}\right], \ldots, G_{M}=\left[L_{M}, \infty\right)$. On each $G_{j}$ there is a $k_{j}, 1 \leq k_{j} \leq d-1$, such that for $r \in G_{j}$ we have $|P(r)| \sim\left|b_{k_{j}}\right| r^{k_{j}}$ and $\left|P^{\prime}(r)\right| \sim\left|b_{k_{j}}\right| r^{k_{j}-1}$.

For each $j, 1 \leq j \leq M$, consider the (possibly empty) "dyadic" interval $D_{j}$ defined as

$$
D_{j}=\left[\delta\left|b_{k_{j}} / b_{d}\right|^{1 / d-k_{j}}, C\left|b_{k_{j}} / b_{d}\right|^{1 / d-k_{j}}\right] \cap G_{j},
$$

where $\delta$ and $C$ will be chosen to depend only on $d$. The dyadic intervals for $Q$ are now defined to be the dyadic intervals for $P$, together with $\left\{D_{j}\right\}_{j=1}^{M}$. It is clear from the construction that (1) and (i), (ii) of (2) hold for $Q$. To see that the pointwise bounds for $Q$ and $Q^{\prime}$ hold it suffices to note that the gaps for $Q$ are formed by splitting each gap for $P$ into at most two intervals. If $I=\left[\delta\left|b_{k_{j}} / b_{d}\right|^{1 / d-k_{j}}, C\left|b_{k_{j}} / b_{d}\right|^{1 / d-k_{j}}\right]$ is completely contained in $G_{j}$ then $G_{j}$ splits into two gaps, $G_{j_{1}}=\left[L_{j}, \delta\left|b_{k_{j}} / b_{d}\right|^{1 / d-k_{j}}\right]$ and $G_{j_{2}}=$ $\left[C\left|b_{k_{j}} / b_{d}\right|^{1 / d-k_{j}}, R_{j}\right]$. Otherwise, depending on whether $I$ meets $G_{j}$ (or not), $G_{j}$ splits into one gap (or none) for $Q$.

Hence choosing $\delta$ small enough and $C$ large enough, we then have $|Q(r)|$ $\sim\left|b_{k_{j}}\right| r^{k_{j}}$ and $\left|Q^{\prime}(r)\right| \sim\left|b_{k_{j}}\right| r^{k_{j}-1}$ on $G_{j_{1}}$, and $|Q(r)| \sim\left|b_{d}\right| r^{d}$ and $\left|Q^{\prime}(r)\right| \sim$ $\left|b_{d}\right| r^{d-1}$ on $G_{j_{2}}$.

Lemma $2.2([5])$. Let $K(x)=\Omega(x) /|x|^{n}$ be as in Theorem 1.1 and $P(x)$ $=\sum_{|\alpha|=d} c_{\alpha} x^{\alpha}$ be a homogeneous polynomial of degree $d$ on $\mathbb{R}^{n}$. Write $m_{P}=$ $\sum_{|\alpha|=d}\left|c_{\alpha}\right|$. Then

$$
\int_{\mathbf{S}^{n-1}}|\Omega(\omega)| \cdot\left|\log \left(\frac{P(\omega)}{m_{P}}\right)\right| d \sigma(\omega) \lesssim 1 .
$$

Proof. We sketch a proof for completeness. We may assume $m_{P}=1$. The part of the integral in (4) where $|P(\omega)|^{-\delta} \leq|\Omega(\omega)|$ (for any $\delta>0$ ) is easily controlled by the $L \log L$ norm of $\Omega$ and so matters are reduced to 
estimating

$$
\int_{\left\{\omega:|\Omega(\omega)| \leq|P(\omega)|^{-\delta}\right\}}|\Omega(\omega)| \cdot|\log (|P(\omega)|)| d \sigma(\omega) .
$$

This integral is controlled by

$$
\begin{aligned}
& \int_{\mathbf{S}^{n-1}}|P(\omega)|^{-\delta}|\log (|P(\omega)|)| d \sigma(\omega) \\
& \lesssim \int_{1 / 2}^{1} r^{n-1} \int_{\mathbf{S}^{n-1}}|P(\omega)|^{-\delta}|\log (|P(\omega)|)| d \sigma(\omega) d r \\
& \lesssim \sum_{k \geq 1} k 2^{\delta k} \int_{1 / 2}^{1} r^{n-1} d r \quad \int_{\left\{\omega \in \mathbf{S}^{n-1}:|P(\omega)| r^{d} \leq 2^{-k}\right\}} d \sigma(\omega) \\
& \leq \sum_{k \geq 1} k 2^{\delta k}\left|\left\{|x| \leq 1:|P(x)| \leq 2^{-k}\right\}\right|,
\end{aligned}
$$

which reduces matters to obtaining uniform sublevel set estimates for $P$ under the normalisation $m_{P}=1$. Using the fact that all norms are equivalent on the space of polynomials of degree at most $d$, we can find a derivative $\partial^{\alpha}, 0 \leq|\alpha| \leq d$, such that $1 \lesssim\left|\partial^{\alpha} P(x)\right|$ on $|x| \leq 1$. If $\alpha=0$, then the above sublevel sets are empty for large $k$ and so we may assume $|\alpha|>0$. In this case, using the mean value theorem, one can show that $\mid\{|x| \leq 1$ : $\left.|P(x)| \leq 2^{-k}\right\} \mid \lesssim 2^{-k /|\alpha|}$ (see e.g. [1]). This establishes the lemma if we choose $\delta<1 / d$.

The last lemma is a very useful estimate for one-dimensional oscillatory integrals, known as van der Corput's lemma. A proof can be found in [5].

LEMMA 2.3. Suppose $\phi$ is real-valued and smooth on $(a, b)$, and that $\left|\phi^{(k)}(t)\right| \geq \lambda>0$ for all $t \in(a, b)$. Then

$$
\left|\int_{a}^{b} e^{i \phi(t)} d t\right| \leq C_{k} \lambda^{-1 / k}
$$

when either $k \geq 2$, or $k=1$ and $\phi^{\prime}(t)$ is monotonic.

3. Proof of Theorem 1.1. We may assume $P(0)=0$. Using polar coordinates write the integral in (3) as

$$
I=\int_{\mathbf{S}^{n-1}} \Omega(\omega) \int_{0}^{\infty} e^{i\left[1 / Q_{\omega}(r)+P_{\omega}(r)\right]} \frac{1}{r} d r d \sigma(\omega)
$$

where $Q(x)=Q_{\omega}(r)=\sum_{j=1}^{d^{\prime}} q_{j}(\omega) r^{j}, P(x)=P_{\omega}(r)=\sum_{k=1}^{d} p_{k}(\omega) r^{k}$ and $q_{j}, p_{k}$ are homogeneous polynomials of degree $j$ and $k$. Using Lemma 2.1, 
we may write $I=\sum I_{j, k}+O(1)$ where

$$
I_{j, k}=\int_{\mathbf{S}^{n-1}} \Omega(\omega) \int_{G_{j} \cap F_{k}} e^{i\left[1 / Q_{\omega}(r)+P_{\omega}(r)\right]} \frac{1}{r} d r d \sigma(\omega) .
$$

Here $\left\{G_{j}\right\}$ and $\left\{F_{k}\right\}$ are the "gaps" of $Q_{\omega}(r)$ and $P_{\omega}(r)$ respectively. Note that although the inner integral of $I_{j, k}$ depends on $\omega$ in a complicated way, we know the form of the endpoints of $G_{j}$ and $F_{k}$ as given by Lemma 2.1 and so it is at least measurable as a function of $\omega$. It suffices to bound each $I_{j, k}$ separately.

We have $\left|Q_{\omega}(r)\right| \sim\left|q_{j_{l}}(\omega)\right| r^{j_{l}}$ and $\left|Q_{\omega}^{\prime}(r)\right| \sim\left|q_{j_{l}}(\omega)\right| r^{j_{l}-1}$ on $G_{j}$, for some $1 \leq j_{l} \leq d^{\prime}$, and $\left|P_{\omega}(r)\right| \sim\left|p_{k_{m}}(\omega)\right| r^{k_{m}}$ and $\left|P_{\omega}^{\prime}(r)\right| \sim\left|p_{k_{m}}(\omega)\right| r^{k_{m}-1}$ on $F_{k}$ for some $1 \leq k_{m} \leq d$.

Therefore away from where $r^{j_{l}+k_{m}} \sim\left(\left|p_{k_{m}}(\omega)\right| \cdot\left|q_{j_{l}}(\omega)\right|\right)^{-1}$ the size of the phase $\phi_{\omega}(r)=P_{\omega}(r)+1 / Q_{\omega}(r)$ and its derivative is understood. In fact, on $R_{j, k}=G_{j} \cap F_{k} \cap\left[C\left(\left|p_{k_{m}}(\omega)\right| \cdot\left|q_{j_{l}}(\omega)\right|\right)^{-1 /\left(k_{m}+j_{l}\right)}, \infty\right)$ (for $C$ large enough), we have

$$
\left|\phi_{\omega}(r)\right| \sim\left|p_{k_{m}}(\omega)\right| r^{k_{m}} \quad \text { and } \quad\left|\phi_{\omega}^{\prime}(r)\right| \sim\left|p_{k_{m}}(\omega)\right| r^{k_{m}-1} .
$$

An application of van der Corput's Lemma 2.3 shows

$$
\int_{\left\{r \in R_{j, k}: r \geq \Theta\right\}} e^{i \phi_{\omega}(r)} \frac{1}{r} d r=O(1)
$$

where $\Theta=\left|p_{k_{m}}(\omega)\right|^{-1 / k_{m}}$. Since we are applying Lemma 2.3 with $k=1$, we need to first split the integration of the above integral into $O(1)$ intervals where $\phi_{\omega}^{\prime}(r)$ is monotone. In the complementary interval, $r \leq \Theta$, due to the size of $\phi_{\omega}(r)$ on $R_{j, k}$, we see that

$$
\int_{\left\{r \in R_{j, k}: r \leq \Theta\right\}}\left[e^{i \phi_{\omega}(r)}-1\right] \frac{d r}{r}=O(1) .
$$

Therefore for the part of $I_{j, k}$ over $R_{j, k}$,

$$
\int_{\mathbf{S}^{n-1}} \Omega(\omega) \int_{R_{j, k}} e^{i \phi_{\omega}(r)} \frac{1}{r} d r d \sigma(\omega)=\int_{\mathbf{S}^{n-1}} \Omega(\omega) \int_{\left\{r \in R_{j, k}: r \leq \Theta\right\}} \frac{d r}{r} d \sigma(\omega)+O(1) .
$$
have

Similarly for $L_{j, k}=G_{j} \cap F_{k} \cap\left(-\infty, \delta\left(\left|p_{k_{m}}(\omega)\right| \cdot\left|q_{j_{l}}(\omega)\right|\right)^{-1 /\left(k_{m}+j_{l}\right)}\right]$, we

$$
\int_{\mathbf{S}^{n-1}} \Omega(\omega) \int_{L_{j, k}} e^{i \phi_{\omega}(r)} \frac{1}{r} d r d \sigma(\omega)=\int_{\mathbf{S}^{n-1}} \Omega(\omega) \int_{\left\{r \in L_{j, k}: r \geq \Lambda\right\}} \frac{d r}{r} d \sigma(\omega)+O(1)
$$

where $\Lambda=\left|q_{j_{l}}(\omega)\right|^{-1 / j_{l}}$. Therefore 


$$
\begin{aligned}
I_{j, k}= & \int_{\mathbf{S}^{n-1}} \Omega(\omega) \int_{G_{j} \cap F_{k}} e^{i\left[P_{\omega}(r)+1 / Q_{\omega}(r)\right]} \frac{1}{r} d r d \sigma(\omega) \\
= & \int_{\mathbf{S}^{n-1}} \Omega(\omega) \int_{\left\{r \in R_{j, k}: r \leq \Theta\right\}} \frac{d r}{r} d \sigma(\omega) \\
& +\int_{\mathbf{S}^{n-1}} \Omega(\omega) \int_{\left\{r \in L_{j, k}: r \geq \Lambda\right\}} \frac{d r}{r} d \sigma(\omega)+O(1)
\end{aligned}
$$

and these two last integrals can be shown to be $O(1)$ by repeatedly applying Lemma 2.2.

In fact, the integrals in (5) are of the form

$$
\int_{\mathbf{S}^{n-1}} \Omega(\omega) \int_{E(\omega)} \frac{d r}{r} d \sigma(\omega)
$$

where $E(\omega)$ is the intersection of $O(1)$ intervals of the form $[a(\omega), \infty)$ or $(-\infty, a(\omega)]$ where

$$
\begin{array}{r}
a(\omega) \in\left\{\left(\left|p_{k_{1}}(\omega)\right| /\left|p_{k_{2}}(\omega)\right|\right)^{1 /\left(k_{2}-k_{1}\right)},\left(\left|q_{j_{1}}(\omega)\right| /\left|q_{j_{2}}(\omega)\right|\right)^{1 /\left(j_{2}-j_{1}\right)},\right. \\
\left.\left|p_{k}(\omega)\right|^{-1 / k},\left|q_{j}(\omega)\right|^{-1 / j},\left(\left|q_{j}(\omega)\right| \cdot\left|p_{k}(\omega)\right|\right)^{-1 /(j+k)}\right\} .
\end{array}
$$

Let us write $E(\omega)=[a(\omega), \infty) \cap E^{\prime}(\omega)$ where $a(\omega)$, say, is $\left(\left|q_{j}(\omega)\right|\right.$. $\left.\left|p_{k}(\omega)\right|\right)^{-1 /(j+k)}$ and $E^{\prime}(\omega)$ is the intersection of one less intervals. We can then use Lemma 2.2 to write

$$
\int_{\mathbf{S}^{n-1}} \Omega(\omega) \int_{E(\omega)} \frac{d r}{r} d \sigma(\omega)=\int_{\mathbf{S}^{n-1}} \Omega(\omega) \int_{[A, \infty) \cap E^{\prime}(\omega)} \frac{d r}{r} d \sigma(\omega)+O(1)
$$

where $A=\left(m_{q_{j}} m_{p_{k}}\right)^{-1 /(j+k)}$. In fact,

$$
\begin{aligned}
& \int_{\mathbf{S}^{n-1}} \Omega(\omega) \int_{[a(\omega), \infty) \cap E^{\prime}(\omega)} \frac{d r}{r} d \sigma(\omega) \\
& =\int_{\mathbf{S}^{n-1}} \Omega(\omega) \int_{[A, \infty) \cap E^{\prime}(\omega)} \frac{d r}{r} d \sigma(\omega)-\int_{\mathbf{S}^{n-1}} \Omega(\omega) \int_{[A, a(\omega)] \cap E^{\prime}(\omega)} \frac{d r}{r} d \sigma(\omega)
\end{aligned}
$$

and the second integral is $O(1)$ by Lemma 2.2. Iterating this $O(1)$ times (the argument is similar for the other forms of $a(\omega)$ ) reduces the problem to an integral of the form

$$
\int_{\mathbf{S}^{n-1}} \Omega(\omega) \int_{E} \frac{d r}{r} d \sigma(\omega)
$$

where $E$ no longer depends on $\omega$ and so this integral vanishes from the mean value of $\Omega$ being zero. This concludes the proof of Theorem 1.1. 
4. Proof of Proposition 1.4. Recall that now $Q(x)=a+v \cdot x$. Again we write the integral (3) in polar coordinates

$$
\int_{\mathbf{S}^{n-1}} \Omega(\omega) \int_{0}^{\infty} e^{i\left(P_{\omega}(r)+1 /[a+(v \cdot \omega) r]\right)} \frac{1}{r} d r d \sigma(\omega)
$$

where we continue to use the notation $P_{\omega}(r)$ as before. There are just two "gaps" for $Q$ now: $(0, \delta|a| /|v \cdot \omega|]$ and $[C|a| /|v \cdot \omega|, \infty)$. The difficulty occurs when the $r$ integration is restricted to the first gap. The argument over the second gap is the same as before and so we will only concern ourselves with the first gap; let us call this interval $G$. If we denote by $\phi_{\omega}(r)$ the phase as before, then on $G$,

$$
\left|\phi_{\omega}^{(k)}(r)\right| \sim|v \cdot \omega|^{k} /|a|^{k+1}
$$

for any $k$ strictly larger than the degree of $P$. Fix such a $k$. We may apply van der Corput's lemma to see that

$$
\int_{\mathbf{S}^{n-1}} \Omega(\omega) \int_{\left\{r \in G: r \geq \Theta_{k}\right\}} e^{i \phi_{\omega}(r)} \frac{1}{r} d r d \sigma(\omega)=O(1)
$$

where $\Theta_{k}=|a|^{1+1 / k} /|v \cdot \omega|$. Hence we may assume $|a|<1$ and restrict ourselves to the subinterval $G^{\prime}=\left(0, \Theta_{k}\right]$ of $G$. Considering the Taylor expansion of $1 /[a+(v \cdot \omega) r]$ in $r$, we see that on $G$ (and hence on $G^{\prime}$ ),

$$
\phi_{\omega}(r)=\widetilde{P}_{\omega}(r)+O\left(\frac{|v \cdot \omega|^{k}}{|a|^{k+1}} r^{k}\right)
$$

where $\widetilde{P}(x)$ is some polynomial of degree at most $k$. Thus

$$
\int_{\mathbf{S}^{n-1}} \Omega(\omega) \int_{G^{\prime}}\left[e^{i \phi_{\omega}(r)}-e^{i \widetilde{P}_{\omega}(r)}\right] \frac{1}{r} d r d \sigma(\omega)=O(1)
$$

and the proof of Proposition 1.4 is reduced to showing that

$$
\int_{\mathbf{S}^{n-1}} \Omega(\omega) \int_{G^{\prime}} e^{i \widetilde{P}_{\omega}(r)} \frac{1}{r} d r d \sigma(\omega)=\int_{|x \cdot v| \leq N} \exp (i \widetilde{P}(x)) K(x) d x
$$

is uniformly bounded in $v, N$ and the coefficients of $\widetilde{P}$ (here $N=|a|^{1+1 / k}$ ). We may assume $|v|=1$. By (1),

$$
\int_{|x| \leq N} \exp (i \widetilde{P}(x)) K(x) d x=O(1)
$$

which reduces the problem to estimating

$$
\int_{N \leq|x|,|x \cdot v| \leq N} \exp (i \widetilde{P}(x)) K(x) d x
$$

But this is trivially bounded as $K$ is uniformly integrable over this region. This completes the proof of Proposition 1.4. 


\section{References}

[1] A. Carbery, M. Christ and J. Wright, Multidimensional van der Corput and sublevel set estimates, J. Amer. Math. Soc. 12 (1999), 981-1015.

[2] A. Carbery, F. Ricci and J. Wright, Maximal functions and Hilbert transforms associated to polynomials, Rev. Mat. Iberoamericana 14 (1998), 117-144.

[3] F. Ricci and E. M. Stein, Harmonic analysis on nilpotent groups and singular integrals I: Oscillatory integrals, J. Funct. Anal. 73 (1987), 179-194.

[4] - - - Harmonic analysis on nilpotent groups and singular integrals II: Singular kernels supported on submanifolds, ibid. 78 (1988), 56-84.

[5] E. M. Stein (ed.), Beijing Lectures in Harmonic Analysis, Ann. of Math. Stud. 112, Princeton Univ. Press, 1986.

[6] E. M. Stein and S. Wainger, The estimation of an integral arising in multiplier transformations, Studia Math. 35 (1970), 101-104.

Instituto de Matemáticas, UNAM

Area de la Investigación Científica

Circuito Exterior, Ciudad Universitaria

México, D.F. 04510, México

E-mail: folchgab@matem.unam.mx
Department of Mathematics and Statistics University of Edinburgh JCMB, King's Buildings Mayfield Road Edinburgh EH9 3JZ, Scotland E-mail: wright@maths.ed.ac.uk

Received February 4, 2002

Revised version June 10, 2002 